\title{
INSULIN AND ANTI INFLAMMATION: AN EFFECT BEYOND GLYCEMIC CONTROL
}

Madhav Prabhu' ${ }^{1}$ V A Kothiwale ${ }^{2}$

\section{HOW TO CITE THIS ARTICLE:}

Madhav Prabhu, VA Kothiwale. "Insulin and anti inflammation: an effect beyond glycemic control". Journal of Evolution of Medical and Dental Sciences 2013; Vol2, Issue 43, October 28; Page: 8232-8239.

ABSTRACT: Choosing between OHA and insulin especially in type 2 diabetes has always been a challenge. Recent studies have indicated that insulin proved superior as it has additional inflammatory properties. Thus insulin could prove beneficial especially in diabetics with coronary artery disease (CAD) where inflammation causes plaque instability. With this background we studied the influence of insulin on inflammation in patients with coronary artery disease. The influence of admission sugars and duration of disease was also studied. METHODS: 83 diabetics coming with history of diabetes were studied .Patients were divided into those on insulin and those on OHA. Admission RBS and HsCRP (inflammatory marker) were done after a thorough history. Appropriate statistical methods were employed where necessary. RESULTS: HsCRP was consistently higher in patients with higher admission RBS ( $p=.000)$, however patients on insulin had comparatively low HsCRP levels despite high RBS values ( $p=.000)$. Mean HsCRP values were higher in patients with myocardial ischemia and unstable angina than in stable angina, hereto it was observed that patients on insulin had much lower HsCRP values ( $\mathrm{p}=.005)$. HsCRP was highest in those with diabetes of less than 1yr duration ( $p=000)$. HsCRP was much lower in insulin group irrespective of the duration of therapy $(\mathrm{p}=.010)$. CONCLUSION: Insulin exerts marked antiinflammatory effect on patients with CAD irrespective of admission sugars and duration of diabetes. Insulin could thus add to plaque stability and improved outcomes in patients with CAD.

KEY WORDS: HsCRP, Insulin, Coronary Artery Disease

INTRODUCTION: Diabetes is the most common metabolic disorder all over the world. Diabetes has now reached pandemic proportions; it has been recognized as an independent risk factor for various diseases including the dreaded ischemic heart diseases. Recently the role of inflammation in the pathogenesis of diabetic complications has been recognized. Inflammation which is now recognized as a key mechanism in diabetes also contributes to increased atherosclerosis and plaque instability which can cause many an unstable coronary syndromes. Therapy in diabetes has always been a subject of intense debate. An oral hypoglycemic drug (OHA) versus insulin as the option of choice in the treatment of diabetes has always been a dilemma. Recent studies have demonstrated that in sulin has a marked anti-inflammatory effect in addition to its action of glycemic control ${ }^{1-4}$. Thus insulin could score a point over OHAs as anti inflammation would confer additional protective effect especially in ischemic heart disease. With this background in mind we conducted this study to evaluate the anti-inflammatory effect of insulin in patients of diabetes coming with coronary artery disease using HsCRP as a marker of inflammation.

METHODS: Patient Selection-we included 83 patients coming to KLE Hospital Belgaum with history of coronary artery disease including chronic stable angina (SA), Unstable Angina(USA) and myocardial infarction(MI). Patients were interviewed at admission according to a predetermined 
questionnaire after an informed written consent. Diagnosis was established based on history, electrophysiology and serum enzymes in accordance with standard diagnostic criteria. History of diabetes with mention to the time from onset, the type of treatment and the duration of treatment was elicited. All our study subjects were known diabetes; all were on the same therapy that is either insulin or OHA for a minimum of 6 months before the inclusion into the study. All patients with renal or hepatic diseases, autoimmune disorders and chronic inflammatory diseases were excluded as these conditions could influence HsCRP levels and give false values. We also excluded patients with hypertension, smoking and other risk factors for coronary artery disease as this could influence HsCRP levels. Patients with fever and history of surgery in the past three months were also excluded.

Laboratory assessment-once consent was taken patients blood sample was collected for cardiac enzymes, HsCRP and other biochemical parameters. Random sugars were measured at admission. Samples for HsCRP were collected and sent to laboratory as soon as possible .Patients serum HsCRP was measured using PETIA(Particle Enhanced Turbidimetric Immuno analysis Method) using Dade Behring UK kit. HsCRP values were reported in $\mathrm{mg} / \mathrm{L}$. The assay had sensitivity to detect as low as $0.5 \mathrm{mg} /$ liter of CRP. Undetectable CRP values were recorded as $0.015 \mathrm{mg} / \mathrm{liter}$. Levels greater than 1 were considered significant.

Statistical methods-statistical analysis was done using standard statistical methods. ANOVA test was used for correlation of HsCRP with admission RBS and duration of disease. T-test was used to compare HsCRP in types of treatment. Univariate analysis using general linear mode was used to compare disease types with type and duration of treatment with HsCRP. Other statistical methods were employed wherever necessary.

RESULTS: Of the 83 patients in our study there were 27 with unstable angina (USA), 17 with stable angina (SA) and 39 with myocardial infarction (MI). In the study group 39 were on insulin and 44 were on OHAs. Most patients came with admission sugars of 200-300 mg/dl. HsCRP showed a linear relation with blood sugars and was highest in patients with highest sugars with a high correlation coefficient of $p=.000$.HsCRP was significantly higher in OHA group in comparison with insulin group irrespective of the admission RBS ( $\mathrm{p}=.010$ ) see table 1.

Mean HsCRP was significantly higher in OHA group (8.59) than in the insulin group(4.25) ,this was statistically significant $p=.000$.HsCRP and thus the inflammatory burden correlated significantly with the type of disease with the mean HsCRP much higher in unstable diseases like unstable angina and myocardial infarction than in stable angina this was statistically significant. In all the disease types it was noticed that the insulin group had significantly lower HsCRP than the OHA group which was also statistically significant $(\mathrm{p}=.000)$ as shown in table 2 .

HsCRP and thus inflammation did not show any significant relation with the duration of therapy $(p=0.632)$. However it was noticed that irrespective of the duration of therapy HsCRP was much lower in the insulin group than the OHA group ( $\mathrm{p}=.010)$ as shown in table 3.

Therapy: Most patients in our study had a history of diabetes for one to five years. Thus HsCRP was significantly lower in insulin group when compared to OHA group.

DISCUSSION: In the study we found that insulin showed anti-inflammatory effect irrespective of the glycemic status of the individuals. There are two possible mechanisms for the anti-inflammatory 
effects of insulin one being its ability to control blood sugars which add to the inflammatory burden and the second being an inherent anti inflammatory effect of insulin.

Pro-inflammatory effects of glucose and response to insulin: There have been several studies that have demonstrated the proinflammatory effects of glucose. This may then mean that the antiinflammatory effect of insulin may be secondary to its glucose lowering effect. Glucose has been shown to induce the generation of ROS and superoxide radical and to induce P47phox by leucocytes upon the administration of $75 \mathrm{~g}$ of glucose even in normal subject's normal subject's ${ }^{5}$.Thus blood sugars can increase the oxidative stress even in the presence of a non diabetic state. Glucose intake was proved to cause translocation of P47phox to the cell membrane and also increasing NF $\mathrm{k} \beta$ (regulator of inflammation) binding and the activation of IkB kinases a and b with a decrease in IkBa6. Glucose intake also increases tumor necrosis factor a (TNF $\alpha$ ) mRNA. This was shown in one study where infusion of glucose into normal subjects results in an increase in TNF $\alpha$ and IL- 6 if the subject's endogenous insulin secretion is inhibited by the concomitant infusion of somatostatin ${ }^{7}$. In one study glucose intake has also been shown to increase activator protein-1 (AP-1) and Egr-1, two major pro-inflammatory transcription factors and the key genes activated by them: MMP-2, MMP-9 and tissue factor (TF) ${ }^{8}$. TF is an activator of the extrinsic pathway of coagulation; this may explain the predisposition of patients with poor glycemic status to develop thrombosis 9. Glucose has been shown to increase thrombin activation in subjects with type 2 diabetes and this effect has been reduced with the administration of antioxidants. Thus glucose itself may be a potent stimulator of inflammatory activity. This adds proof to the theory that it may be the sugar lowering by medications or life style modification may contribute to anti-inflammation. In our study we demonstrated that the admission glycemic status did have a marked effect on the inflammatory burden in an individual. It was also noticed that the inflammatory burden was much lower in patients who were on insulin rather than in tho se who were on OHA across the spectrum of glycemic control. It was also noticed that the inflammatory burden in patients was low even in those patients with high blood sugars; this means that insulin irrespective of the sugars has an ability to bring down the inflammatory burden which may be induced by blood glucose. As OHAs did not demonstrate any such effects it may be reasonable to conclude that insulin may be superior to OHA at least in its anti-inflammatory effect. This benefit is relevant more so in intensive care set ups where stress related increase in sugars may cause greater damage here ant inflammation by insulin may be an absolute necessity. One study we found that insulin therapy with its anti-inflammatory effect could explains the beneficial effects on organ failure and mortality in medically and surgical critically ill patients. ${ }^{10}$ Our study also demonstrated this benefit.

Insulin as an anti-inflammatory agent: The next prospect that would also need attention would be whether insulin itself is an anti inflammatory molecule. Several studies have shown that insulin suppresses the expression of the pro-inflammatory intracellular adhesion molecule (ICAM)-1, the chemokine, monocyte chemoattractant protein-1 (MCP-1), and the key pro-inflammatory transcription factor, nuclear factor $\mathrm{k} \beta(\mathrm{NF} \mathrm{k} \beta$ ) in human tissue cells 1, 2, 3. Insulin also was shown to causes an acute reduction in plasma concentrations of ICAM-1, MCP-1and another pro-inflammatory transcription factor, early growth response-1 (Egr-1), tissue factor and plasminogen activator inhibitor-1 (PAI-1) 11. In recent experiments intensive insulin treatment during sepsis was shown to decrease proinflammatory (IL-1, IL-6, macrophage inflammatory factor, TNF $\alpha$ ) and increase antiinflammatory cytokines (IL-4, IL-10) (9), indicating an immunomodulating effect. One study has 
shown that insulin influences $\mathrm{T}$ cell differentiation, promoting a shift toward a Th2-type response rather than a Th1type of response which alters the Th1 to Th2 ratio and thus reducing inflammation by reducing cell mediated immune response ${ }^{12}$. Insulin has also been shown to suppress matrix metalloproteinase (MMP)-9 and vascular endothelial growth factor (VEGF), two key mediators involved in the spread of inflammation and in the increase of vascular permeability 13, 14. Recently data demonstrating interference by insulin on signal transduction of interleukin-6 (IL-6) on adipocytes, in vitro, has also been shown. Insulin has been shown to inhibit IL-6 induced phosphorylation and activation of STAT-3 which leads to its translocation into the nucleus and the transcriptional activation of genes regulated by STAT-3 which include SAA and haptoglobin 15. Furthermore, anti apoptotic effects of insulin and other growth factors have also been described, and inhibiting sepsis-induced apoptosis of $\mathrm{T}$ and $\mathrm{B}$ cells is thought to be beneficial. Thus these studies demonstrate that insulin in itself may have a marked anti-inflammatory effect. Our study demonstrated this anti-inflammatory effect and also a fact that this effect starts as soon as the therapy is started that is nearly one year and is sustained over a long period of time. Insulin early in the treatment of diabetes has always been a subject of intense debate the above evidence would support early insulin over OHA for its anti inflammatory effect.

Anti inflammation by insulin and Cardio protection: Hyperglycemia has always been found to be associated with poorer outcome in patients with strokes, myocardial infarction and several other acute conditions. Inflammation has been known to contribute to this poor outcome. Inflammation causes plaque instability and thus more episodes of unstable coronary syndromes like unstable angina and myocardial infarctions. In our study too we found that patients with unstable coronary diseases had more amount of inflammation. We also found that patients with unstable diseases who were on insulin therapy had inflammatory burdens much lower than those on OHAs. Several studies have demonstrated such benefits. In one study subjects with ST-segment elevation myocardial infarction, undergoing percutaneous coronary intervention, and a blood glucose of over160 mg/dl increased the risk of no reflow phenomenon, and a blood glucose of over $140 \mathrm{mg} / \mathrm{dl}$ was associated with a 2.6-fold increase in the failure of spontaneous reperfusion 16, 17. This could possibly be due to the proinflammatory effect of blood glucose and insulin therapy would be of help here. In another study insulin infusion titrated to reduce blood glucose has been shown to improve clinical outcomes in hyperglycemic patients even in those who were non diabetics hospitalized with an AMI 18, 19. Insulin has also been shown to reduce the myocardial infarct size, independent of FFA modulation in experimental MI 20, 21, 22. In these models, insulin infused prior to reperfusion has reduced the size of the myocardial infarct by nearly $45 \%$.The potential benefits of insulin could be secondary to its antiinflammatory effects. In another study involving patients with acute myocardial infarction, insulin was also shown to suppress C-reactive protein (CRP) and serum amyloid A (SAA) by $40 \%$ within 24 $\mathrm{h}$ of the initiation of the insulin infusion even though glucose concentrations were not allowed to change ${ }^{23}$. Treatment of acute myocardial infarction (AMI) patients with insulin has been shown to suppress the inflammatory molecules PAI-1, pro-MMP-1 23, 24. In patients treated in an ICU, insulin infusions have also been shown to suppress inducible nitric oxide synthase (iNOS) expression in the liver and reduce plasma concentrations of nitrite and nitrate, the two metabolites of nitric oxide 25. In addition to the above anti-inflammatory effect, insulin has also been shown to induce vasodilatation in arteries and veins and to induce increased microcirculatory (capillary) flow 26, 27. This effect is mediated by the stimulation of eNOS and the generation of endothelial nitric oxide 
leading to an increase in cGMP in the vascular smooth muscle ${ }^{26,27 . ~ I n s u l i n ~ h a s ~ a l s o ~ b e e n ~ s h o w n ~ t o ~}$ exert an antiplatelet effect, in vitro and in vivo ${ }^{28}$. This effect is mediated by platelet nitric oxide synthase activation and the release of nitric oxide and the subsequent generation of cGMP from guanylate cyclase ${ }^{29}$. This effect has recently been shown to occur in patients with acute coronary syndrome following an intravenous infusion of insulin ${ }^{30}$. Such an antiplatelet effect of insulin would contribute further to its anti-inflammatory effect since the platelet is loaded with CD40 ligand which when bound to its receptor, CD40 expressed on leucocytes and endothelial cells, activates proinflammatory pathways ${ }^{31}$. The inhibition of platelet aggregation would thus prevent the activation of a major pro-inflammatory pathway in addition to suppressing thrombotic tendencies which is one of the aims of therapy in these patients. Thus insulin by several novel mechanisms would be cardioprotective especially in settings of acute coronary settings.

CONCLUSION: Inflammation plays a critical role in the pathogenesis of several acute conditions including sepsis, stroke and myocardial infarction. Hyperglycemic is a potent proinflammatory stimulus, which may explain the poorer prognosis in patients with raised sugars. Insulin plays a critical role in diabetic therapy especially in acute settings. Insulin has a marked anti-inflammatory effect; this may be due to its glucose lowering effects and also through potent inherent antiinflammatory properties. Insulin reduces anti-inflammatory burden soon after initiation of therapy and this effect is sustained over a long period. The additional cardioprotective effects of insulin would make it a agent of choice in further therapeutic strategies.

\section{The authors of these paper have no conflicts of interest.}

\section{REFERENCES:}

1. Aljada A, Saadeh R, Assian E, et al. Insulin inhibits the expression of intercellular adhesion molecule- 1 by human aortic endothelial cells through stimulation of nitric oxide. J Clin Endocrinol Metab 2000; 85:2572-2575.

2. Aljada A, Dandona P. Effect of insulin on human aortic endothelial nitric oxide synthase. Metabolism 2000; 49:147-150.

3. Aljada A, Ghanim H, Saadeh R, Dandona P. Insulin inhibits NF kappa B and MCP-1 expression in human aortic endothelial cells. J Clin Endocrinol Metab 2001; 86:450-453.

4. Dandona P, Aljada A, Mohanty $\mathrm{P}$, et al. Insulin inhibits intranuclear nuclear factor kappa B and stimulates I kappa B in mononuclear cells in obese subjects: evidence for an antiinflammatory effect? J Clin Endocrinol Metab 2001; 86:3257-3265.

5. Mohanty P, Hamouda W, Garg R, et al. Glucose challenge stimulates reactive oxygen species (ROS) generation by leucocytes. J Clin Endocrinol Metab 2000; 85:2970-2973.

6. Dhindsa S, Tripathy D, Mohanty P, et al. Differential effects of glucose and alcohol on reactive oxygen species generation and intranuclear nuclear factor-kappa B in mononuclear cells. Metabolism 2004; 53:330-334.

7. Esposito K, Nappo F, Marfella R, et al. Inflammatory cytokine concentrations are acutely increased by hyperglycemia in humans: role of oxidative stress. Circulation 2002; 106:20672072. 
8. Aljada A, Ghanim H, Mohanty $\mathrm{P}$, et al. Glucose intake induces an increase in activator protein 1 and early growth response 1 binding activities, in the expression of tissue factor and matrix metalloproteinase in mononuclear cells, and in plasma tissue factor and matrix metalloproteinase concentrations. Am J Clin Nutr 2004; 80:51-57.

9. Mackman N. Role of tissue factor in hemostasis, thrombosis, and vascular development. Arterioscler Thromb Vasc Biol 2004; 24:1015-1022.

10. Troels Krarup Hansen, Steffen Thiel, Pieter Jozef Wouyers,et al. Intensive Insulin Therapy Exerts Antiinflammatory Effects in Critically Ill Patients and Counteracts the Adverse Effect of Low Mannose-Binding Lectin Levels The Journal of Clinical Endocrinology \& Metabolism 88(3):1082-1088doi: 10.1210/jc.2002-021478.

11. Aljada A, Ghanim H, Mohanty $\mathrm{P}$, et al. Insulin inhibits the pro-inflammatory transcription factor early growth response gene-1 (Egr)-1 expression in mononuclear cells (MNC) and reduces plasma tissue factor (TF) and plasminogen activator inhibitor-1 (PAI-1) concentrations. J Clin Endocrinol Metab 2002; 87:1419-1422.

12. Alexander Viardot, Shane T. Grey, Fabienne Mackay and Donald Chisholm. Potential Antiinflammatory Role of Insulin via the Preferential Polarization of Effector T Cells toward a T Helper 2 Phenotype: Endocrinology 148(1):346-353doi: 10.1210/en.2006-0686.

13. Dandona P, Aljada A, Mohanty P, et al. Insulin suppresses plasma concentration of vascular endothelial growth factor and matrix metalloproteinase-9. Diabetes Care 2003; 26:33103314.

14. Weis S, Shintani S, Weber A, et al. Src blockade stabilizes a Flk/cadherin complex, reducing edema and tissue injury following myocardial infarction. J Clin Invest 2004; 113:885-894.

15. Andersson CX, Sopasakis VR, Wallerstedt E, Smith U. Insulin antagonizes IL- 6 signaling and is anti-inflammatory in 3T3-L1 adipocytes. J Biol Chem 2007; 282:9430-9435.

16. Iwakura $\mathrm{K}$, Ito $\mathrm{H}$, Ikushima $\mathrm{M}$, et al. Association between hyperglycemia and the no-reflow phenomenon in patients with acute myocardial infarction. J AmColl Cardiol 2003; 41:1-7.

17. Timmer JR, Ottervanger JP, de Boer MJ, et al. Hyperglycemia is an important predictor of impaired coronary flow before reperfusion therapy in ST-segment elevation myocardial infarction. J Am Coll Cardiol 2005; 45:999-1002.

18. Cheung NW, Wong VW, McLean M. The Hyperglycemia: Intensive Insulin Infusion in Infarction (HI-5) study: a randomized controlled trial of insulin infusion therapy for myocardial infarction. Diabetes Care 2006; 29:765-770.

19. Malmberg K, Ryden L, Efendic S, et al. Randomized trial of insulin-glucose infusion followed by subcutaneous insulin treatment in diabetic patients with acute myocardial infarction (DIGAMI study): effects on mortality at 1 year. J Am Coll Cardiol 1995; 26:57-65.

20. Gao F, Gao E, Yue TL, et al. Nitric oxide mediates the antiapoptotic effect of insulin in myocardial ischemia-reperfusion: the roles of PI3-kinase, Akt, and endothelial nitric oxide synthase phosphorylation. Circulation 2002; 105:1497-1502.

21. Jonassen AK, Brar BK, Mjos OD, et al. Insulin administered at reoxygenation exerts a cardioprotective effect in myocytes by a possible antiapoptotic mechanism. J Mol Cell Cardiol 2000; 32:757-764. 
22. Zhang HX, Zang YM, Huo JH, et al. Physiologically tolerable insulin reduces myocardial injury and improves cardiac functional recovery in myocardial ischemic/reperfused dogs. J Cardiovasc Pharmacol 2006; 48:306-313.

23. Chaudhuri A, Janicke D, Wilson MF, et al. Anti-inflammatory and profibrinolytic effect of insulin in acute ST-segment-elevation myocardial infarction. Circulation 2004; 109:849-854.

24. Chaudhuri A, Janicke D, Wilson MF, et al. Free fatty acid suppressive, Pro-MMP-1 lowering and cardioprotective effect of insulin in STEMI. Diabetes 2005; 54:

25. Langouche L, Vanhorebeek I, Vlasselaers D, et al. Intensive insulin therapy protects the endothelium of critically ill patients. J Clin Invest 2005; 115:2277-2286.

26. Grover A, Padginton C, Wilson MF, et al. Insulin attenuates norepinephrine induced venoconstriction. An ultrasonographic study. Hypertension 1995; 25:779-784.

27. Steinberg HO, Brechtel G, Johnson A, et al. Insulin-mediated skeletal muscle vasodilation is nitric oxide dependent. A novel action of insulin to increase nitric oxide release. J Clin Invest 1994; 94:1172-1179.

28. Trovati M, Massucco P, Mattiello L, et al. Insulin increases guanosine-30, 50-cyclic monophosphate in human platelets. A mechanism involved in the insulin anti aggregating effect. Diabetes 1994; 43:1015-1019.

29. Trovati M, Anfossi G, Massucco P, et al. Insulin stimulates nitric oxide synthesis in human platelets and, through nitric oxide, increases platelet concentrations of both guanosine-30, 50-cyclic monophosphate and adenosine-30, 50-cyclic monophosphate. Diabetes 1997; 46:742-749.

30. Worthley MI, Holmes AS, Willoughby SR, et al. The deleterious effects of hyperglycemia on platelet function in diabetic patients with acute coronary syndromes: mediation by superoxide production, resolution with intensive insulin administration. J Am Coll Cardiol 2007; 49:304-310.

31. Libby P, Simon DI. Inflammation and thrombosis: the clot thickens. Circulation 2001; 103:1718-1720.

\begin{tabular}{|c|c|c|c|c|}
\hline RBS mg/dl & $\mathbf{n =}$ & Mean HsCRP (mg/lt) & $\begin{array}{c}\text { INSULIN } \\
\text { GROUP (mg/lt) }\end{array}$ & $\begin{array}{c}\text { OHA } \\
\text { GROUP(mg/lt) }\end{array}$ \\
\hline $\mathbf{1 0 0 - 2 0 0}$ & 25 & 4.78 & 3.90 & 7.57 \\
\hline $\mathbf{2 0 1 - 3 0 0}$ & 39 & 5.59 & 4.57 & 6.67 \\
\hline $\mathbf{3 0 1 - 4 0 0}$ & 13 & 10.22 & & 10.22 \\
\hline $\mathbf{> 4 0 0}$ & 6 & 12.16 & & 12.16 \\
\hline Total & 83 & 6.55 & & \\
\hline \multicolumn{5}{|c|}{ Table1- Mean RBS and HsCRP in different glycemic groups } \\
\hline
\end{tabular}

$\mathrm{R}=0.617, \mathrm{p}=.000$ for hsCRP with RBS $\mathrm{p}=.010$ for OHA vs insulin group 


\begin{tabular}{|c|c|c|c|c|}
\hline Diagnose & $\mathbf{n}=$ & Mean HsCRP(mg/lt) & $\begin{array}{c}\text { OHA group } \\
\text { (mg/lt) }\end{array}$ & $\begin{array}{c}\text { Insulin group } \\
\text { (mg/lt) }\end{array}$ \\
\hline SA & 17 & 4.47 & 5.61 & 3.66 \\
\hline USA & 27 & 6.16 & 8.45 & 3.69 \\
\hline MI & 39 & 7.73 & 9.58 & 5.07 \\
\hline \multicolumn{5}{|r}{ Table2- HsCRP in different disease groups } \\
\hline
\end{tabular}

$\mathrm{F}_{2,80}=5.965 \mathrm{P}=.004$ for diagnose $\mathrm{F}=48.525 \mathrm{p}=.000$ for treatment with diagnose

\begin{tabular}{|c|c|c|c|c|}
\hline Duration & $\mathrm{n}=$ & Mean HsCRP (mg/lt) & $\begin{array}{c}\text { Insulin group } \\
(\mathrm{mg} / \mathrm{lt})\end{array}$ & $\begin{array}{c}\text { OHA group } \\
(\mathrm{mg} / \mathrm{lt})\end{array}$ \\
\hline$<\mathbf{1 y r}$ & 18 & 8.58 & 5.59 & 11.57 \\
\hline $\mathbf{1 - 5 y r}$ & 40 & 5.74 & 3.54 & 7.37 \\
\hline$>\mathbf{5 y r}$ & 25 & 6.37 & 4.25 & 8.69 \\
\hline \multicolumn{3}{|r|}{ Table 3- Duration of therapy and HsCRP } \\
\hline
\end{tabular}

$\mathrm{R}=.053, \mathrm{p}=.632$ for duration and HsCRP $\mathrm{f}=64.868, \mathrm{p}=.010$ for duration and type of therapy

\section{AUTHORS:}

1. Madhav Prabhu

2. V.A. Kothiwale

\section{PARTI CULARS OF CONTRIBUTORS:}

1. Assistant Professor, Department of Medicine, Jawaharlal Nehru Medical College, Nehru Nagar, Belgaum, Karnataka, India.

2. Professor, Department of Medicine, Jawaharlal Nehru Medical College, Nehru Nagar, Belgaum, Karnataka, India.

\section{NAME ADDRESS EMAIL ID OF THE}

\section{CORRESPONDING AUTHOR:}

Dr. Madhav Prabhu, Assistant Professor, Departm ent of Medicine, Jawaharlal Nehru Medical College, Nehru Nagar, Bel gaum, Karnataka, India. Email-wiseman2380@yahoo.com

Date of Submission: 14/06/2013. Date of Peer Review: 14/06/2013. Date of Acceptance: 21/06/2013. Date of Publishing: 22/10/2013 